Bulletin of the Section of Logic

Volume 47/2 (2018), pp. 69-88

http://dx.doi.org/10.18778/0138-0680.47.2.01

George Voutsadakis

\title{
CATEGORICAL ABSTRACT ALGEBRAIC LOGIC: PSEUDO-REFERENTIAL MATRIX SYSTEM SEMANTICS
}

\author{
To the memory of A.I. Mal'cev, \\ 50 years since his passing.
}

\begin{abstract}
This work adapts techniques and results first developed by Malinowski and by Marek in the context of referential semantics of sentential logics to the context of logics formalized as $\pi$-institutions. More precisely, the notion of a pseudoreferential matrix system is introduced and it is shown how this construct generalizes that of a referential matrix system. It is then shown that every $\pi$-institution has a pseudo-referential matrix system semantics. This contrasts with referential matrix system semantics which is only available for self-extensional $\pi$-institutions by a previous result of the author obtained as an extension of a classical result of Wójcicki. Finally, it is shown that it is possible to replace an arbitrary pseudoreferential matrix system semantics by a discrete pseudo-referential matrix system semantics.

Keywords: Referential Logics, Selfextensional Logics, Referential Semantics, Referential $\pi$-institutions, Selfextensional $\pi$-institutions, PseudoReferential Semantics, Discrete Referential Semantics.
\end{abstract}

2010 AMS Subject Classification: 03G27

\section{Introduction}

Let $\mathcal{L}=\langle\Lambda, \rho\rangle$ be a logical signature/algebraic type, i.e., a set of logical connectives/operation symbols $\Lambda$ with attached finite arities given by the 
function $\rho: \Lambda \rightarrow \omega$. Let also $V$ be a countably infinite set of propositional variables and $T$ a set of reference/base points. Wójcicki [10] defines a referential algebra $\boldsymbol{A}$ over $T$ (or based on $T$ ) to be an $\mathcal{L}$-algebra with universe $A \subseteq\{0,1\}^{T}$, or, equivalently, $A \subseteq \mathcal{P}(T)$.

Let $\mathbf{F m}_{\mathcal{L}}(V)=\left\langle\operatorname{Fm}_{\mathcal{L}}(V), \mathcal{L}\right\rangle$ be the free $\mathcal{L}$-algebra generated by the set $V$ of variables. A homomorphism from $\mathbf{F m}_{\mathcal{L}}(V)$ into a referential algebra $\boldsymbol{A}$ over $T$ may be viewed as an interpretation of the formulas of $\operatorname{Fm}_{\mathcal{L}}(V)$ in $A$. We conceive of a formula $\alpha \in \mathrm{Fm}_{\mathcal{L}}(V)$ as being true at point $t \in T$ under $h$ if and only if $t \in h(\alpha)$. This notion of truth gives rise to a consequence operation on $\operatorname{Fm}_{\mathcal{L}}(V)$. Namely, a referential algebra $\boldsymbol{A}$ determines the consequence operator $C^{\boldsymbol{A}}$ on $\operatorname{Fm}_{\mathcal{L}}(V)$ by setting, for all $X \cup\{\alpha\} \subseteq \operatorname{Fm}_{\mathcal{L}}(V), \alpha \in C^{\boldsymbol{A}}(X)$ iff, for all $h: \mathbf{F m}_{\mathcal{L}}(V) \rightarrow \boldsymbol{A}$ and all $t \in T$,

$$
h(\beta)(t)=1 \text {, for all } \beta \in X, \text { implies } h(\alpha)(t)=1,
$$

or equivalently, iff, for all $h: \mathbf{F m}_{\mathcal{L}}(V) \rightarrow \boldsymbol{A}$,

$$
\bigcap_{\beta \in X} h(\beta) \subseteq h(\alpha) .
$$

Wójcicki calls a propositional logic $\mathcal{S}=\langle\mathcal{L}, C\rangle$, where $C=C^{\boldsymbol{A}}$, for a referential algebra $\boldsymbol{A}$, a referential (or referentially truth-functional) propositional logic.

Wójcicki shows in [10] that, given a class $\mathrm{K}$ of referential algebras, there exists a single referential algebra $\boldsymbol{A}$, such that $C^{\mathrm{K}}:=\bigcap_{\boldsymbol{K} \in \mathrm{K}} C^{\boldsymbol{K}}=C^{\boldsymbol{A}}$. Thence follows that a propositional logic is referential if and only if it is defined by a class of referential algebras.

Given a propositional logic $\mathcal{S}=\langle\mathcal{L}, C\rangle$, the Frege or interderivability relation of $\mathcal{S}$, denoted $\Lambda(\mathcal{S})$, is the equivalence relation on $\operatorname{Fm}_{\mathcal{L}}(V)$, defined, for all $\alpha, \beta \in \operatorname{Fm}_{\mathcal{L}}(V)$, by

$$
\langle\alpha, \beta\rangle \in \Lambda(\mathcal{S}) \quad \text { iff } \quad C(\alpha)=C(\beta) .
$$

The Tarski congruence $\widetilde{\Omega}(\mathcal{S})$ of $\mathcal{S}$ (see [5]) is the largest congruence relation on $\mathbf{F m}_{\mathcal{L}}(V)$ that is compatible with all theories of $\mathcal{S}$. The Tarski congruence is a special case of the Suszko congruence $\widetilde{\Omega}^{\mathcal{S}}(T)$ associated with a given theory $T$ of $\mathcal{S}$, which is defined as the largest congruence on $\mathbf{F m}_{\mathcal{L}}(V)$ that is compatible with all theories of $\mathcal{S}$ that contain the given theory $T$ (see [3]). In fact, by definition, $\widetilde{\Omega}(\mathcal{S})=\widetilde{\Omega}^{\mathcal{S}}(C(\varnothing)$ ), i.e., the Tarski congruence of $\mathcal{S}$ is the Suszko congruence associated with the set of 
theorems of the logic $\mathcal{S}$. Font and Jansana [5], extending Czelakowski's [2] (see also [1]) well-known characterization of the Leibniz congruence $\Omega(T)$ associated with a theory $T$ of a sentential logic, have shown that, for all $\alpha, \beta \in \operatorname{Fm}_{\mathcal{L}}(V)$,

$$
\begin{array}{ll}
\langle\alpha, \beta\rangle \in \widetilde{\Omega}(\mathcal{S}) \quad \text { iff } \quad \text { for all } \varphi(p, \vec{q}) \in \operatorname{Fm}_{\mathcal{L}}(V), \\
& C(\varphi(\alpha, \vec{q}))=C(\varphi(\beta, \vec{q})) .
\end{array}
$$

Whereas $\widetilde{\Omega}(\mathcal{S}) \subseteq \Lambda(\mathcal{S})$, for every propositional logic $\mathcal{S}$, the reverse inclusion does not hold in general. A propositional logic is called selfextensional in $[10]$ if $\Lambda(\mathcal{S}) \subseteq \widetilde{\Omega}(\mathcal{S})$. In fact, Wójcicki shows in what has become a fundamental theorem in the theory of referential semantics, Theorem 2 of [10], that a propositional logic is referential if and only if it is selfextensional. This result shows that, unless a propositional logic $\mathcal{S}$ is selfextensional, $\mathcal{S}$ cannot possess a referential algebraic semantics.

Let $\mathcal{L}$ be a logical signature. An $\mathcal{L}$-g-matrix $\mathbb{A}=\langle\boldsymbol{A}, \mathcal{C}\rangle$ consists of an $\mathcal{L}$-algebra $\boldsymbol{A}$ together with a collection $\mathcal{C} \subseteq \mathcal{P}(A)$. A g-matrix $\mathbb{A}$ generates a consequence operator $C^{\mathbb{A}}$ on $\operatorname{Fm}_{\mathcal{L}}(V)$ as follows: For all $X \cup\{\alpha\} \subseteq \operatorname{Fm}_{\mathcal{L}}(V)$,

$$
\begin{aligned}
\alpha \in C^{\mathbb{A}}(X) \quad \text { iff } & \text { for all } h: \mathbf{F m}_{\mathcal{L}}(V) \rightarrow \boldsymbol{A} \text { and all } F \in \mathcal{C}, \\
& h(X) \subseteq F \operatorname{implies} h(\alpha) \in F .
\end{aligned}
$$

A g-matrix $\mathbb{A}$ is said to constitute a g-matrix semantics for a propositional logic $\mathcal{S}=\langle\mathcal{L}, C\rangle$ in case $C^{\mathbb{A}}=C$.

Consider now a referential algebra $\boldsymbol{A}$ over a set $T$ of reference points. Let, for all $t \in T$,

$$
D_{t}=\{a \in A: t \in a\} .
$$

Define the collection $\mathcal{D}=\left\{D_{t}: t \in T\right\}$. We call $\langle\boldsymbol{A}, \mathcal{D}\rangle$ the referential g-matrix associated with the referential algebra $\boldsymbol{A}$.

It can be shown that the consequence operator $C^{\langle\boldsymbol{A}, \mathcal{D}\rangle}$ generated by the g-matrix system $\langle\boldsymbol{A}, \mathcal{D}\rangle$ is identical to $C^{\boldsymbol{A}}$. Thus, it follows that, unless $\mathcal{S}$ is self-extensional it does not possess a referential g-matrix semantics.

To address this shortcoming of referential g-matrices in providing a semantics for arbitrary propositional logics, Malinowski introduced in [8] pseudo-referential g-matrices, as a generalization of referential g-matrices, and showed that every propositional logic possesses a pseudo-referential g-matrix semantics.

Let, once more, $T$ be a set of reference points and consider, also, a collection $T^{*} \subseteq \mathcal{P}(T)$ of subsets of $T$. According to [8] a pseudo-referential 
g-matrix $\mathbb{A}=\langle\boldsymbol{A}, \mathcal{D}\rangle$ relative to $\left(T, T^{*}\right)$ is a g-matrix, such that $\boldsymbol{A}$ is a referential algebra based on $T$ and

$$
\begin{aligned}
\mathcal{D} & =\left\{\left\{a \in A:\left(\exists t \in t^{*}\right)(t \in a)\right\}: t^{*} \in T^{*}\right\} \\
& =\left\{\left\{a \in A: a \cap t^{*} \neq \varnothing\right\}: t^{*} \in T^{*}\right\} .
\end{aligned}
$$

Note that this concept generalizes referentiality, since a referential g-matrix associated with a referential algebra $\boldsymbol{A}$ based on $T$ is obtained as a special case of a pseudo-referential g-matrix relative to $\left(T, T^{*}\right)$, with $T^{*}=\{\{t\}$ : $t \in T\}$.

In the Theorem of [8] it is shown that every propositional logic $\mathcal{S}$ has a strongly adequate pseudo-referential g-matrix $\mathbb{A}$, which may be termed the canonical pseudo-referential g-matrix associated with $\mathcal{S}$.

Malinowski's work was followed by Marek [9]. Marek defines a discrete pseudo-referential g-matrix as a pseudo-referential g-matrix relative to a pair $\left(T, T^{*}\right)$, such that $T^{*} \subseteq\{\{t\}: t \in T\}$. She then shows that every g-matrix is isomorphic to, and, hence, generates the same sentential logic as, a discrete pseudo-referential g-matrix. Thus, since, as is well-known, every propositional logic has a strongly adequate g-matrix semantics, it follows that it also has a strongly adequate discrete pseudo-referential g-matrix semantics (see Corollary of [9]).

The author, taking after the work of Wójcicki, showed in previous work $[11,12]$ that a logic formalized as a $\pi$-institution (see Section 2 ) is referential, i.e., has a referential g-matrix system semantics, if and only if it is selfextensional. Thus, it turns out that, similarly to the case of propositional logics, for these logics, unless the condition of self-extensionality is fulfilled, no referential g-matrix system semantics is available. The present work, inspired by the previously mentioned work of Malinowski [8] and Marek [9], addresses this constraint on the availability of a referential g-matrix system semantics by introducing a pseudo-referential g-matrix system semantics (see Section 4). It is shown in Theorem 5 that every $\pi$-institution possesses a pseudo-referential g-matrix system semantics. Finally, improving on this result, we show in Section 7, in a parallel to the Theorem of Marek [9], that, for every g-matrix system, there exists a discrete pseudoreferential g-matrix system that generates the same closure system (see Theorem 6). It then follows that every logic formalized as a $\pi$-institution has a discrete pseudo-referential g-matrix semantics. 


\section{2. $\pi$-Institutions and Closure Systems}

We describe $\pi$-institutions [4] (see, also [6] for the closely related notion of an institution) on which our logical systems will be based.

Let Sign $^{b}$ be a category, called the category of signatures. Let $\mathrm{SEN}^{b}: \mathbf{S i g n}^{b} \rightarrow$ Set be a set-valued functor, called the sentence functor. Let $N^{b}$ be a category of natural transformations on $\mathrm{SEN}^{b}$ (see Section 2 of [12]). We call the triple $\boldsymbol{A}^{b}=\left\langle\mathbf{S i g n}^{b}, \mathrm{SEN}^{b}, N^{b}\right\rangle$ the base algebraic system.

A collection $T^{b}=\left\{T_{\Sigma}^{b}\right\}_{\Sigma \epsilon\left|\mathbf{S i g n}^{b}\right|}$, such that $T_{\Sigma}^{b} \subseteq \operatorname{SEN}^{b}(\Sigma)$, for all $\Sigma \epsilon$ $\left|\mathbf{S i g n}^{b}\right|$, is called a sentence family of $\boldsymbol{A}^{b}$.

A $\pi$-institution based on $\boldsymbol{A}^{\mathrm{b}}$ is a pair $\mathcal{I}=\left\langle\boldsymbol{A}^{\mathrm{b}}, C\right\rangle$, where

$$
C=\left\{C_{\Sigma}\right\}_{\Sigma \in\left|\mathbf{S i g n}^{b}\right|}
$$

is a closure (operator) system, i.e., a $\left|\mathbf{S i g n}^{b}\right|$-indexed collection of closure operators $C_{\Sigma}: \mathcal{P}\left(\operatorname{SEN}^{b}(\Sigma)\right) \rightarrow \mathcal{P}\left(\operatorname{SEN}^{b}(\Sigma)\right)$ that satisfy the structurality condition:

For all $\Sigma_{1}, \Sigma_{2} \in\left|\operatorname{Sign}^{b}\right|, f \in \operatorname{Sign}^{b}\left(\Sigma_{1}, \Sigma_{2}\right)$ and $\Phi \subseteq \operatorname{SEN}^{b}\left(\Sigma_{1}\right)$,

$$
\operatorname{SEN}^{b}(f)\left(C_{\Sigma_{1}}(\Phi)\right) \subseteq C_{\Sigma_{2}}\left(\operatorname{SEN}^{b}(f)(\Phi)\right) .
$$

For $\Sigma \in\left|\mathbf{S i g n}^{b}\right|$, a set $T_{\Sigma}^{b} \subseteq \operatorname{SEN}^{b}(\Sigma)$ is called a $\Sigma$-theory of $\mathcal{I}$ if it is closed under consequence, i.e., if $C_{\Sigma}\left(T_{\Sigma}^{b}\right)=T_{\Sigma}^{b}$. The collection of all $\Sigma$-theories of $\mathcal{I}$ is denoted by $\operatorname{Th}_{\Sigma}(\mathcal{I})$. A collection $T^{b}=\left\{T_{\Sigma}^{b}\right\}_{\Sigma \in\left|\operatorname{Sign}^{b}\right|}$, such that $T_{\Sigma}^{b} \in \operatorname{Th}_{\Sigma}(\mathcal{I})$, for all $\Sigma \in\left|\mathbf{S i g n}^{b}\right|$, is called a theory family of $\mathcal{I}$. The collection of all theory families of $\mathcal{I}$ is denoted by $\operatorname{ThFam}(\mathcal{I})$. It is wellknown that they form a complete lattice under signature-wise inclusion $\leq$, whose meet coincides with signature-wise intersection.

Note that closure systems on $\boldsymbol{A}^{\mathrm{b}}$ are ordered as follows:

$$
\begin{aligned}
C^{1} \leq C^{2} \quad \text { iff } \quad \text { for all } & \Sigma \in\left|\mathbf{S i g n}^{b}\right|, \Phi \subseteq \operatorname{SEN}^{b}(\Sigma), \\
& C_{\Sigma}^{1}(\Phi) \subseteq C_{\Sigma}^{2}(\Phi) .
\end{aligned}
$$

Under this ordering the collection of all closure systems on $\boldsymbol{A}^{b}$ also forms a complete lattice whose meet is given by signature-wide intersection.

Given a base algebraic system $\boldsymbol{A}^{b}=\left\langle\mathbf{S i g n}^{b}, \mathrm{SEN}^{b}, N^{b}\right\rangle$, an $N^{b}$-algebraic system $\boldsymbol{A}=\langle\operatorname{Sign}, \mathrm{SEN}, N\rangle$ is an algebraic system, such that there exists a surjective functor $N^{b} \rightarrow N$ preserving all projection natural transformations and, as a consequence, also all the arities of the natural transformations 
involved. We denote by $\sigma: \mathrm{SEN}^{k} \rightarrow \mathrm{SEN}$ the natural transformation that is the image of $\sigma^{b}:\left(\mathrm{SEN}^{b}\right)^{k} \rightarrow \mathrm{SEN}^{b}$ in $N^{b}$, an, in general use similar typing conventions to keep track of mappings of natural transformations in $N^{b}$ to those on $N^{b}$-algebraic systems.

An interpreted $N^{b}$-algebraic system is a pair $\mathcal{A}=\langle\boldsymbol{A},\langle F, \alpha\rangle\rangle$, where

- $\boldsymbol{A}$ is an $N^{\mathrm{b}}$-algebraic system and

- $\langle F, \alpha\rangle: \boldsymbol{A}^{b} \rightarrow \boldsymbol{A}$ is an algebraic system morphism.

We will use the term algebraic system to refer to both an $N^{b}$-algebraic system and an interpreted $N^{b}$-algebraic system relying on the context to clear the ambiguity.

Let $\boldsymbol{A}^{b}$ be an algebraic system and $\mathcal{I}=\left\langle\boldsymbol{A}^{b}, C\right\rangle$ a $\pi$-institution based on $\boldsymbol{A}^{b}$. We define, next, the notion of a matrix system and of a g-matrix system for $\boldsymbol{A}^{b}$ and of a matrix system model and g-matrix system model for $\mathcal{I}$.

A matrix system for $\boldsymbol{A}^{b}$ is a pair $\mathfrak{A}=\langle\mathcal{A}, T\rangle$, where $\mathcal{A}=\langle\boldsymbol{A},\langle F, \alpha\rangle\rangle$ is an interpreted algebraic system and $T$ is a sentence family of $\mathcal{A}$.

A matrix system $\mathfrak{A}$ defines a closure system $C^{\mathfrak{A}}$ (and hence a $\pi$-institution $\left.\mathcal{I}^{\mathfrak{A}}=\left\langle\boldsymbol{A}^{\mathrm{b}}, C^{\mathfrak{A}}\right\rangle\right)$ on $\boldsymbol{A}^{\mathrm{b}}$ as follows: For all $\Sigma \in\left|\mathbf{S i g n}^{\mathrm{b}}\right|$ and all $\Phi \cup\{\varphi\} \subseteq$ $\operatorname{SEN}^{b}(\Sigma)$

$$
\varphi \in C_{\Sigma}^{\mathfrak{A}}(\Phi) \quad \text { iff } \quad \Phi \vDash_{\Sigma}^{\mathfrak{A}} \varphi,
$$

where the relation on the right means that, for all $\Sigma^{\prime} \in\left|\mathbf{S i g n}^{b}\right|$ and all $f \in \operatorname{Sign}^{b}\left(\Sigma, \Sigma^{\prime}\right)$,

$$
\alpha_{\Sigma^{\prime}}\left(\operatorname{SEN}^{b}(f)(\Phi)\right) \subseteq T_{F\left(\Sigma^{\prime}\right)} \quad \text { implies } \quad \alpha_{\Sigma^{\prime}}\left(\operatorname{SEN}^{b}(\varphi)\right) \in T_{F\left(\Sigma^{\prime}\right)} .
$$

A generalized matrix system for $A^{\text {b }}$ (or g-matrix system, for short) is a pair $\mathbb{A}=\langle\mathcal{A}, \mathcal{T}\rangle$, where $\mathcal{A}=\langle\boldsymbol{A},\langle F, \alpha\rangle\rangle$ is an interpreted algebraic system and $\mathcal{T}$ is a collection of sentence families of $\mathcal{A}$.

A g-matrix system $\mathbb{A}$ defines a closure system $C^{\mathbb{A}}$ (and hence a $\pi$-institution $\left.\mathcal{I}^{\mathbb{A}}=\left\langle\boldsymbol{A}^{\mathrm{b}}, C^{\mathbb{A}}\right\rangle\right)$ on $\boldsymbol{A}^{\mathrm{b}}$ by setting $C^{\mathbb{A}}=\bigcap_{\mathfrak{A} \in \mathbb{A}} C^{\mathfrak{A}}$, where $\mathfrak{A}=\langle\boldsymbol{A}, T\rangle \in \mathbb{A}$ means that $T \in \mathcal{T}$. Thus, equivalently, for all $\Sigma \in\left|\mathbf{S i g n}^{b}\right|$ and all $\Phi \cup\{\varphi\} \subseteq$ $\operatorname{SEN}^{b}(\Sigma)$,

$$
\varphi \in C_{\Sigma}^{\mathfrak{A}}(\Phi) \quad \text { iff } \quad(\forall \mathfrak{A} \in \mathbb{A})\left(\Phi \vDash_{\Sigma}^{\mathfrak{A}} \varphi\right) .
$$

A matrix system model for $\mathcal{I}=\left\langle A^{b}, C\right\rangle$ or an $\mathcal{I}$-matrix system is a matrix system $\mathfrak{A}=\langle\mathcal{A}, T\rangle$ for $\boldsymbol{A}^{\mathrm{b}}$, such that $C \leq C^{\mathfrak{A}}$.

Similarly, a g-matrix system model for $\mathcal{I}$ or an $\mathcal{I}$-g-matrix system is a g-matrix system $\mathbb{A}$, such that $C \leq C^{\mathbb{A}}$. 


\section{Referential $\pi$-Institutions}

In this work we focus on a special kind of (interpreted) $N^{\mathrm{b}}$-algebraic system $\mathcal{A}=\langle\boldsymbol{A},\langle F, \alpha\rangle\rangle, \boldsymbol{A}=\langle\mathbf{S i g n}, \mathrm{SEN}, N\rangle$. We require that, for all $\Sigma \in|\mathbf{S i g n}|$, there is a set $\operatorname{PTS}(\Sigma)$, called the set of $\Sigma$-reference or $\Sigma$-base points, and that, for all $\Sigma \in|\operatorname{Sign}|, \operatorname{SEN}(\Sigma) \subseteq \mathcal{P}(\operatorname{PTS}(\Sigma))$, i.e., each $\Sigma$-sentence is a set of $\Sigma$-points.

In this context, an interpretation $\langle F, \alpha\rangle: \boldsymbol{A}^{b} \rightarrow \boldsymbol{A}$ will be viewed as a valuation of sentences of $\boldsymbol{A}^{b}$ in the following way: For all $\Sigma \in\left|\mathbf{S i g n}^{b}\right|$ and all $\varphi \in \operatorname{SEN}^{b}(\Sigma), \varphi$ is true at $p \in \operatorname{PTS}(F(\Sigma))$ under $\langle F, \alpha\rangle$ iff $p \in \alpha_{\Sigma}(\varphi)$.

An algebraic system of this special form is called a referential algebraic system and said to be based on PTS.

Note that this definition is a generalized version of the one given in Section 3 of [12]. The generalization stems from the fact that, in the present context, we no longer insist that the sentence functor SEN be a simple subfunctor (having the same domain) of the inverse powerset of a contravariant functor Sign $\rightarrow$ Set $^{\mathrm{op}}$.

Let $\mathcal{A}=\langle\boldsymbol{A},\langle F, \alpha\rangle\rangle$ be an interpreted referential $N^{\mathrm{b}}$-algebraic system. Then $\mathcal{A}$ determines a closure system $C^{\mathcal{A}}$ on $\boldsymbol{A}^{b}$ according to the following definition:

For all $\Sigma \in\left|\operatorname{Sign}^{b}\right|$ and all $\Phi \cup\{\varphi\} \subseteq \operatorname{SEN}^{b}(\Sigma), \varphi \in C_{\Sigma}^{\mathcal{A}}(\Phi)$ iff, for all $\Sigma^{\prime} \in\left|\operatorname{Sign}^{b}\right|$ and all $f \in \operatorname{Sign}^{b}\left(\Sigma, \Sigma^{\prime}\right)$,

$$
\bigcap_{\phi \in \Phi} \alpha_{\Sigma^{\prime}}\left(\operatorname{SEN}^{b}(f)(\phi)\right) \subseteq \alpha_{\Sigma^{\prime}}\left(\operatorname{SEN}(f)^{b}(\varphi)\right) .
$$

Essentially the same proof as that of Proposition 1 of [12] yields the following

Proposition 1 (Proposition 1 of [12]). Let $\boldsymbol{A}^{\mathrm{b}}=\left\langle\operatorname{Sign}^{\mathrm{b}}, \mathrm{SEN}^{\mathrm{b}}, N^{\mathrm{b}}\right\rangle$ be a base algebraic system and $\mathcal{A}=\langle\boldsymbol{A},\langle F, \alpha\rangle\rangle$ an interpreted referential $N^{b}$-algebraic system. Then $C^{\mathcal{A}}$ is a closure system on $\boldsymbol{A}^{\mathrm{b}}$.

Since $C^{\mathcal{A}}$ is a closure system on $\boldsymbol{A}^{\mathrm{b}}$, the pair $\mathcal{I}^{\mathcal{A}}=\left\langle\boldsymbol{A}^{\mathrm{b}}, C^{\mathcal{A}}\right\rangle$ is a $\pi$-institution. We call an institution having this form a referential $\pi$-institution. Such $\pi$-institutions correspond in the theory of categorical abstract algebraic logic to the referential propositional logics of Wójcicki [10].

Let $\boldsymbol{A}^{b}=\left\langle\operatorname{Sign}^{b}, \mathrm{SEN}^{b}, N^{b}\right\rangle$ be a base algebraic system and $\mathcal{I}=\left\langle\boldsymbol{A}^{b}, C\right\rangle$ a $\pi$-institution based on $\boldsymbol{A}^{\mathrm{b}}$. We define the Frege equivalence system 
$\Lambda(\mathcal{I})$ of $\mathcal{I}$, also known as the interderivability equivalence system, by setting, for all $\Sigma \in\left|\operatorname{Sign}^{b}\right|$ and all $\varphi, \psi \in \operatorname{SEN}^{b}(\Sigma)$,

$$
\langle\varphi, \psi\rangle \in \Lambda_{\Sigma}(\mathcal{I}) \quad \text { if and only if } C_{\Sigma}(\varphi)=C_{\Sigma}(\psi) .
$$

The Tarski congruence system $\widetilde{\Omega}(\mathcal{I})$ of $\mathcal{I}$ ([5] for the universal algebraic notion and [13] for its categorical extension) is the largest congruence system on $\boldsymbol{A}^{\mathrm{b}}$ that is compatible with every theory family $T \in \operatorname{ThFam}(\mathcal{I})$.

Clearly, it is always the case that $\widetilde{\Omega}(\mathcal{I}) \leq \Lambda(\mathcal{I})$. We call the $\pi$-institution $\mathcal{I}$ self-extensional if $\Lambda(\mathcal{I}) \leq \widetilde{\Omega}(\mathcal{I})$. In view of the preceding remark, $\mathcal{I}$ is self-extensional if and only if $\Lambda(\mathcal{I})=\widetilde{\Omega}(\mathcal{I})$.

A generalization to $\pi$-institutions of Wójcicki's Theorem (see Theorem 2 of [10], but, also, Theorem 2.2 of [7] for a complete proof), provides a characterization of referential sentential logics. This is essentially Theorem 8 of [12], with the aforementioned generalization pertaining to the signature category not affecting the proof.

TheOREM 2 (Theorem 8 of [12]). A $\pi$-institution $\mathcal{I}=\left\langle\boldsymbol{A}^{\mathrm{b}}, C\right\rangle$ is referential if and only if it is self-extensional.

We recall here a version of the construction of the canonical referential algebraic system associated with a given selfextensional $\pi$-institution that witnesses one implication of Theorem 2.

Let $\mathcal{I}=\left\langle\boldsymbol{A}^{\mathrm{b}}, C\right\rangle$, with $\boldsymbol{A}^{\mathrm{b}}=\left\langle\mathbf{S i g n}^{\mathrm{b}}, \mathrm{SEN}^{\mathrm{b}}, N^{\mathrm{b}}\right\rangle$, be a self-extensional $\pi$-institution. For each $\Sigma \in\left|\mathbf{S i g n}^{b}\right|$, we take as the set of $\Sigma$-points the set $\mathrm{Th}_{\Sigma}(\mathcal{I})$ of $\Sigma$-theories of $\mathcal{I}$.

Define the functor SEN : Sign ${ }^{b} \rightarrow$ Set as follows:

For every $\Sigma \in\left|\mathbf{S i g n}^{b}\right|$,

$$
\operatorname{SEN}(\Sigma)=\left\{\operatorname{Th}_{\Sigma}(\varphi): \varphi \in \operatorname{SEN}^{b}(\Sigma)\right\},
$$

where $\operatorname{Th}_{\Sigma}(\varphi)=\left\{T \in \operatorname{Th}_{\Sigma}(\mathcal{I}): \varphi \in T\right\}$, for all $\Sigma \in\left|\operatorname{Sign}^{b}\right|$ and all $\varphi \epsilon$ $\operatorname{SEN}^{b}(\Sigma)$.

Moreover, for all $\Sigma, \Sigma^{\prime} \in\left|\mathbf{S i g n}^{\mathrm{b}}\right|$, and all $f \in \operatorname{Sign}^{\mathrm{b}}\left(\Sigma, \Sigma^{\prime}\right)$, we define $\operatorname{SEN}(f): \operatorname{SEN}(\Sigma) \rightarrow \operatorname{SEN}\left(\Sigma^{\prime}\right)$ by setting

$$
\operatorname{SEN}(f)\left(\operatorname{Th}_{\Sigma}(\varphi)\right)=\operatorname{Th}_{\Sigma^{\prime}}\left(\operatorname{SEN}^{b}(f)(\varphi)\right)
$$

for all $\Sigma \in\left|\mathbf{S i g n}^{b}\right|$ and all $\varphi \in \operatorname{SEN}^{b}(\Sigma)$.

Define the category of natural transformations $N$ on SEN as follows: For every $\sigma^{b}:\left(\mathrm{SEN}^{b}\right)^{k} \rightarrow \mathrm{SEN}^{b}$ in $N^{b}$, let $\sigma: \mathrm{SEN}^{k} \rightarrow \mathrm{SEN}$ be defined by letting, for all $\Sigma \in\left|\mathbf{S i g n}^{b}\right|, \sigma_{\Sigma}: \operatorname{SEN}(\Sigma)^{k} \rightarrow \operatorname{SEN}(\Sigma)$ be given by 


$$
\sigma_{\Sigma}\left(\operatorname{Th}_{\Sigma}\left(\varphi_{0}\right), \ldots, \operatorname{Th}_{\Sigma}\left(\varphi_{k-1}\right)\right)=\operatorname{Th}_{\Sigma}\left(\sigma_{\Sigma}^{b}\left(\varphi_{0}, \ldots, \varphi_{k-1}\right)\right),
$$

for all $\varphi_{0}, \ldots, \varphi_{k-1} \in \operatorname{SEN}^{b}(\Sigma)$. Using self-extensionality one may show that this is well-defined. Moreover, $\sigma$ is a natural transformation and the collection of natural transformations, thus defined, forms a category of natural transformations on SEN. So the triple $\boldsymbol{A}=\left\langle\mathbf{S i g n}^{b}, \mathrm{SEN}, N\right\rangle$ constitutes an $N^{\text {b}}$-algebraic system.

Finally, the canonical referential algebraic system associated with $\mathcal{I}$ is defined by $\mathcal{A}=\langle\boldsymbol{A},\langle I, \alpha\rangle\rangle$, where:

- $I: \mathbf{S i g n}^{b} \rightarrow \mathbf{S i g n}^{b}$ is the identity functor;

- $\alpha: \mathrm{SEN}^{b} \rightarrow \mathrm{SEN}$ is the natural transformation defined by letting, for all $\Sigma \in\left|\operatorname{Sign}^{b}\right|, \alpha_{\Sigma}: \operatorname{SEN}^{b}(\Sigma) \rightarrow \operatorname{SEN}(\Sigma)$ be given by

$$
\alpha_{\Sigma}(\varphi)=\operatorname{Th}_{\Sigma}(\varphi) \text {, for all } \varphi \in \operatorname{SEN}^{b}(\Sigma) .
$$

Note, now, that, for all $\Sigma, \Sigma^{\prime} \in\left|\mathbf{S i g n}^{b}\right|, f \in \operatorname{Sign}^{b}\left(\Sigma, \Sigma^{\prime}\right)$ and $\varphi \in \operatorname{SEN}^{b}(\Sigma)$,

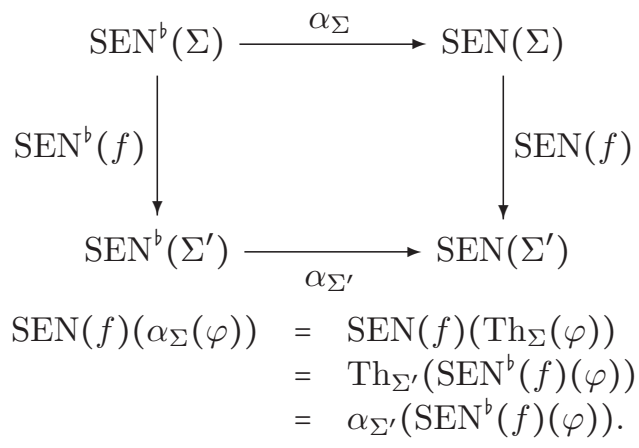

It can be shown that, if $\mathcal{I}$ is self-extensional, then $\mathcal{A}$ is well-defined and, moreover, $\mathcal{I}=\mathcal{I}^{\mathcal{A}}$. Thus, $\mathcal{I}$ is referential.

\section{Pseudo-Referential Matrix Systems}

Let $\boldsymbol{A}^{b}=\left\langle\mathbf{S i g n}^{b}, \mathrm{SEN}^{b}, N^{b}\right\rangle$ be a base algebraic system and $\mathcal{A}=\langle\boldsymbol{A},\langle F, \alpha\rangle\rangle$ a referential $N^{b}$-algebraic system based on PTS. The algebraic system $\mathcal{A}$ will be said to be supported if it is endowed with a collection $\mathcal{S}=\left\{S^{i}: i \in I\right\}$ of base point families

$$
S^{i}=\left\{S_{\Sigma}^{i}\right\}_{\Sigma \epsilon|\operatorname{Sign}|},
$$

where $S_{\Sigma}^{i} \subseteq \operatorname{PTS}(\Sigma)$, for all $i \in I$ and all $\Sigma \in|\operatorname{Sign}|$. We refer to $\mathcal{S}$ as the support of $\mathcal{A}$ in this case. 
Given a supported algebraic system $\mathcal{A}$, with support $\mathcal{S}$, a pseudoreferential g-matrix system relative to $(\mathrm{PTS}, \mathcal{S})$ is a pair

$$
\mathbb{A}=\langle\mathcal{A}, \mathcal{T}\rangle,
$$

where $\mathcal{T}=\left\{T^{i}: i \in I\right\}$ is a collection of sentence families $T^{i}=\left\{T_{\Sigma}^{i}\right\}_{\Sigma \in|\mathbf{S i g n}|}$, such that, for all $i \in I$ and all $\Sigma \in \mid$ Sign $\mid$,

$$
T_{\Sigma}^{i}=\left\{X \in \operatorname{SEN}(\Sigma): X \cap S_{\Sigma}^{i} \neq \varnothing\right\} .
$$

We close this section with two properties of pseudo-referential g-matrix systems. The first states that, in a precise model-theoretic sense, pseudoreferential g-matrix systems encompass referential algebraic systems. The second characterizes the closure system $C^{\mathbb{A}}$ induced by a pseudo-referential g-matrix system on the base algebraic system $\boldsymbol{A}^{\mathrm{b}}$.

Let $\mathcal{A}=\langle\boldsymbol{A},\langle F, \alpha\rangle\rangle$ be a referential algebraic system, based on PTS. Consider the set $\mathcal{P}$ of all $\mid$ Sign|-indexed tuples $P$, such that, for some $\Sigma \in \mid$ Sign $\mid$,

$$
P_{\Sigma^{\prime}} \begin{cases}\epsilon\{\{p\}: p \in \operatorname{PTS}(\Sigma)\}, & \text { if } \Sigma^{\prime}=\Sigma \\ =\varnothing, & \text { if } \Sigma^{\prime} \neq \Sigma\end{cases}
$$

Consider the pseudo-referential g-matrix system $\mathbb{A}(\mathcal{A})=\langle\mathcal{A}, \mathcal{T}\rangle$ relative to (PTS, $\mathcal{P})$. This is called the pseudo-referential g-matrix system associated with $\mathcal{A}$. Then we have the following:

Lemma 3. Let $\boldsymbol{A}^{b}=\left\langle\mathbf{S i g n}^{b}, \mathrm{SEN}^{b}, N^{b}\right\rangle$ be a base algebraic system, $\mathcal{A}=$ $\langle\boldsymbol{A},\langle F, \alpha\rangle\rangle$ a referential $N^{b}$-algebraic system and $\mathbb{A}(\mathcal{A})=\langle\mathcal{A}, \mathcal{T}\rangle$ the pseudoreferential g-matrix system associated with $\mathcal{A}$. Then $C^{\mathcal{A}}=C^{\mathbb{A}(\mathcal{A})}$.

Proof: This follows easily from the fact that, according to the definitions involved, for all $\Sigma \in\left|\mathbf{S i g n}^{b}\right|$ and all $\varphi \in \operatorname{SEN}^{b}(\Sigma)$, we have

$$
p \in \alpha_{\Sigma^{\prime}}\left(\operatorname{SEN}^{b}(f)(\varphi)\right) \text { iff } \alpha_{\Sigma^{\prime}}\left(\operatorname{SEN}^{b}(f)(\varphi)\right) \cap\{p\} \neq \varnothing,
$$

for all $\Sigma^{\prime} \in\left|\mathbf{S i g n}^{b}\right|$, all $f \in \operatorname{Sign}^{b}\left(\Sigma, \Sigma^{\prime}\right)$ and all $p \in \operatorname{PTS}\left(\Sigma^{\prime}\right)$.

Thus, by identifying $\mathcal{A}$ with $\mathbb{A}(\mathcal{A})$ we may view referential algebraic semantics in the sense of [12] as a special case of pseudo-referential g-matrix system semantics.

We now obtain the following characterization of $C^{\mathbb{A}}$ for an arbitrary pseudo-referential g-matrix system $\mathbb{A}$. 
Proposition 4. Let $\boldsymbol{A}^{b}=\left\langle\mathbf{S i g n}^{b}, \mathrm{SEN}^{b}, N^{b}\right\rangle$ be a base algebraic system, $\mathcal{A}=\langle\boldsymbol{A},\langle F, \alpha\rangle\rangle$ an $N^{b}$-referential algebraic system based on $\mathrm{PTS}$, and $\mathbb{A}=$ $\langle\mathcal{A}, \mathcal{T}\rangle$ a pseudo-referential g-matrix system relative to a pair (PTS, $\mathcal{S})$, with $\mathcal{S}=\left\{S^{i}: i \in I\right\}$. Then, for all $\Sigma \in\left|\operatorname{Sign}^{b}\right|, \Phi \cup\{\varphi\} \subseteq \operatorname{SEN}^{b}(\Sigma)$, $\varphi \in C_{\Sigma}^{\mathbb{A}}(\Phi)$ iff, for all $\Sigma^{\prime} \in\left|\mathbf{S i g n}^{\mathrm{b}}\right|, f \in \operatorname{Sign}^{\mathrm{b}}\left(\Sigma, \Sigma^{\prime}\right)$ and all $i \in I$,

$$
\begin{aligned}
\alpha_{\Sigma^{\prime}}\left(\operatorname{SEN}^{b}(f)(\phi)\right) & \cap S_{F\left(\Sigma^{\prime}\right)}^{i} \neq \varnothing, \text { for all } \phi \in \Phi, \\
& \text { implies } \alpha_{\Sigma^{\prime}}\left(\operatorname{SEN}^{b}(f)(\varphi)\right) \cap S_{F\left(\Sigma^{\prime}\right)}^{i} \neq \varnothing .
\end{aligned}
$$

Proof: Let $\Sigma \in\left|\operatorname{Sign}^{b}\right|$ and $\Phi \cup\{\varphi\} \subseteq \operatorname{SEN}^{b}(\Sigma)$. We have $\varphi \in C_{\Sigma}^{\mathbb{A}}(\Phi)$ iff, by definition, for all $\Sigma^{\prime} \in\left|\mathbf{S i g n}^{b}\right|, f \in \mathbf{S i g n}^{b}\left(\Sigma, \Sigma^{\prime}\right)$ and all $i \in I$,

$$
\alpha_{\Sigma^{\prime}}(\operatorname{SEN}(f)(\Phi)) \subseteq T_{F\left(\Sigma^{\prime}\right)}^{i} \quad \text { implies } \quad \alpha_{\Sigma^{\prime}}(\operatorname{SEN}(f)(\varphi)) \in T_{F\left(\Sigma^{\prime}\right)}^{i} .
$$

By the definition of $T^{i}$, this is equivalent to having, for all $\Sigma^{\prime} \in\left|\mathbf{S i g n}^{b}\right|$, $f \in \operatorname{Sign}^{b}\left(\Sigma, \Sigma^{\prime}\right)$ and all $i \in I$,

$$
\begin{aligned}
& \alpha_{\Sigma^{\prime}}\left(\operatorname{SEN}^{b}(f)(\Phi)\right) \subseteq\left\{X \in \operatorname{SEN}\left(F\left(\Sigma^{\prime}\right)\right): X \cap S_{F\left(\Sigma^{\prime}\right)}^{i} \neq \varnothing\right\} \\
& \quad \text { implies } \alpha_{\Sigma^{\prime}}\left(\operatorname{SEN}^{b}(f)(\varphi)\right) \in\left\{X \in \operatorname{SEN}\left(F\left(\Sigma^{\prime}\right)\right): X \cap T_{F\left(\Sigma^{\prime}\right)}^{i} \neq \varnothing\right\} .
\end{aligned}
$$

Equivalently, for all $\Sigma^{\prime} \in\left|\mathbf{S i g n}^{\mathrm{b}}\right|, f \in \mathbf{S i g n}^{\mathrm{b}}\left(\Sigma, \Sigma^{\prime}\right)$ and all $i \in I$,

$$
\begin{aligned}
\alpha_{\Sigma^{\prime}}\left(\operatorname{SEN}^{b}(f)(\phi)\right) \cap S_{F\left(\Sigma^{\prime}\right)}^{i} & \neq \varnothing, \text { for all } \phi \in \Phi, \\
& \text { implies } \alpha_{\Sigma^{\prime}}\left(\operatorname{SEN}^{b}(f)(\varphi)\right) \cap S_{F\left(\Sigma^{\prime}\right)}^{i} \neq \varnothing .
\end{aligned}
$$

\section{Universality of the Semantics}

In this section we show that every $\pi$-institution has a pseudo-referential matrix semantics. This contrasts with Theorem 2, which implies that not every $\pi$-institution has a referential algebraic semantics.

THEOREM 5. Let $\mathcal{I}=\left\langle\boldsymbol{A}^{b}, C\right\rangle$ be a $\pi$-institution based on an algebraic system $\boldsymbol{A}^{b}=\left\langle\operatorname{Sign}^{b}, \mathrm{SEN}^{b}, N^{b}\right\rangle$. Then, there exists a pseudo-referential g-matrix system $\mathbb{A}=\langle\mathcal{A}, \mathcal{T}\rangle$ relative to a pair $(\mathrm{PTS}, \mathcal{S})$, such that $\mathcal{I}=\mathcal{I}^{\mathbb{A}}$, i.e., $C=C^{\mathbb{A}}$.

Proof: Let Sign $=\mathbf{S i g n}^{b}$. For all $\Sigma \in\left|\operatorname{Sign}^{b}\right|$, let $\operatorname{PTS}(\Sigma)=\operatorname{SEN}^{b}(\Sigma)$. Now we define $\boldsymbol{A}=\langle$ Sign, SEN,$N\rangle$ based on PTS as follows: 
- $\operatorname{SEN}(\Sigma)=\left\{\{\varphi\}: \varphi \in \operatorname{SEN}^{b}(\Sigma)\right\}$, for all $\Sigma \in\left|\mathbf{S i g n}^{b}\right|$. And, given, $\Sigma, \Sigma^{\prime} \in\left|\mathbf{S i g n}^{b}\right|, f \in \operatorname{Sign}^{b}\left(\Sigma, \Sigma^{\prime}\right)$,

$$
\operatorname{SEN}(f)(\{\varphi\})=\left\{\operatorname{SEN}^{b}(f)(\varphi)\right\} \text {, for all } \varphi \in \operatorname{SEN}^{b}(\Sigma) \text {. }
$$

- For all $\sigma^{b}:\left(\mathrm{SEN}^{b}\right)^{k} \rightarrow \mathrm{SEN}^{b}$ in $N^{b}$, all $\Sigma \in\left|\mathbf{S i g n}^{b}\right|$ and all $\varphi_{0}, \ldots, \varphi_{k-1} \epsilon$ $\operatorname{SEN}^{b}(\Sigma)^{k}$

$$
\sigma_{\Sigma}\left(\left\{\varphi_{0}\right\}, \ldots,\left\{\varphi_{k-1}\right\}\right)=\left\{\sigma_{\Sigma}^{b}\left(\varphi_{0}, \ldots, \varphi_{k-1}\right)\right\} .
$$

We let $N$ consist of all natural transformations of this form.

It is not difficult to see that, with these definitions, the triple $\boldsymbol{A}=\langle\mathbf{S i g n}$, SEN, $N\rangle$ becomes a referential $N^{b}$-algebraic system based on PTS.

Next, define $\langle I, \alpha\rangle: \boldsymbol{A}^{\mathrm{b}} \rightarrow \boldsymbol{A}$ by setting

- $I: \mathbf{S i g n}^{b} \rightarrow \operatorname{Sign}$ the identity functor;

- For all $\Sigma \in\left|\operatorname{Sign}^{b}\right|$ and all $\varphi \in \operatorname{SEN}^{b}(\Sigma), \alpha_{\Sigma}(\varphi)=\{\varphi\}$.

Now $\mathcal{A}=\langle\boldsymbol{A},\langle I, \alpha\rangle\rangle$ is an interpreted referential $N^{\mathrm{b}}$-algebraic system.

Let $\mathcal{S}=\operatorname{ThFam}(\mathcal{I})=\left\{S^{i}: i \in I\right\}$. This determines the pseudoreferential g-matrix system $\mathbb{A}=\langle\mathcal{A}, \mathcal{T}\rangle$ relative to $(\mathrm{PTS}, \mathcal{S})$. We have that $\mathcal{T}=\left\{T^{i}: i \in I\right\}$, with $T^{i}=\left\{T_{\Sigma}^{i}\right\}_{\Sigma \in|\operatorname{Sign}|}$ given, for all $i \in I$ and all $\Sigma \in|\operatorname{Sign}|$, by

$$
\begin{aligned}
T_{\Sigma}^{i} & =\left\{\{\varphi\} \in \operatorname{SEN}(\Sigma):\{\varphi\} \cap S_{\Sigma}^{i} \neq \varnothing\right\} \\
& =\left\{\{\varphi\} \in \operatorname{SEN}(\Sigma): \varphi \in S_{\Sigma}^{i}\right\},
\end{aligned}
$$

We prove that $C=C^{\mathbb{A}}$, i.e., that, for all $\Sigma \in\left|\mathbf{S i g n}^{b}\right|$ and all $\Phi \cup\{\varphi\} \subseteq$ $\operatorname{SEN}^{b}(\Sigma)$,

$$
\varphi \in C_{\Sigma}(\Phi) \quad \text { iff } \quad \varphi \in C_{\Sigma}^{\mathbb{A}}(\Phi) .
$$

$\Rightarrow$ : Suppose that $\varphi \in C_{\Sigma}(\Phi)$. Let $\Sigma^{\prime} \in\left|\operatorname{Sign}^{b}\right|, f \in \operatorname{Sign}^{b}\left(\Sigma, \Sigma^{\prime}\right)$ and $i \in I$, such that $\alpha_{\Sigma^{\prime}}\left(\operatorname{SEN}^{b}(f)(\phi)\right) \subseteq T_{\Sigma^{\prime}}^{i}$, for all $\phi \in \Phi$. By the definition of $\alpha$, this holds iff $\left\{\operatorname{SEN}^{b}(f)(\phi)\right\} \in T_{\Sigma^{\prime}}^{i}$, for all $\phi \in \Phi$. By the expression given above for $T^{i}$, this holds iff $\operatorname{SEN}^{b}(f)(\phi) \in S_{\Sigma^{\prime}}^{i}$, for all $\phi \in \Phi$, i.e., iff $\operatorname{SEN}^{b}(f)(\Phi) \subseteq S_{\Sigma^{\prime}}^{i}$. Then, since by hypothesis $\varphi \in C_{\Sigma}(\Phi)$, we get $\operatorname{SEN}^{b}(f)(\varphi) \in S_{\Sigma^{\prime}}^{i}$. This shows that $\left\{\operatorname{SEN}^{b}(f)(\varphi)\right\} \in T_{\Sigma^{\prime}}^{i}$ or, equivalently, $\alpha_{\Sigma^{\prime}}\left(\operatorname{SEN}^{b}(f)(\varphi)\right) \in T_{\Sigma^{\prime}}^{i}$. Therefore, $\varphi \in C_{\Sigma}^{\mathbb{A}}(\Phi)$.

$\Leftarrow$ : Suppose that $\varphi \in C_{\Sigma}^{\mathbb{A}}(\Phi)$. Let $i \in I$, such that $\Phi \subseteq S_{\Sigma}^{i}$. This is equivalent to $\{\phi\} \in T_{\Sigma}^{i}$, for all $\phi \in \Phi$. Since, by hypothesis $\varphi \in C_{\Sigma}^{\mathbb{A}}(\Phi)$, 
we get that $\{\varphi\} \in T_{\Sigma}^{i}$. Equivalently, $\varphi \in S_{\Sigma}^{i}$. Since $i \in I$ was arbitrary, we get that $\varphi \in C_{\Sigma}(\Phi)$.

We call the pseudo-referential g-matrix system $\mathbb{A}$, constructed in the proof of Theorem 5 , such that $\mathcal{I}^{\mathbb{A}}=\mathcal{I}$, the canonical pseudo-referential g-matrix system associated with $\mathcal{I}$.

\section{Selfextensional $\pi$-Institutions}

In this section, we start with a selfextensional $\pi$-institution $\mathcal{I}$ and show how, starting from the canonical pseudo-referential g-matrix system associated with $\mathcal{I}$, a process of dividing out by the Frege equivalence system of $\mathcal{I}$ (which is a congruence system due to selfextensionality), leads to the canonical referential g-matrix system for $\mathcal{I}$ constructed in [12]. We present an outline, omitting some of the details that are easy to check.

Let $\mathcal{I}=\left\langle\boldsymbol{A}^{\mathrm{b}}, C\right\rangle$ be a selfextensional $\pi$-institution based on the algebraic system $\boldsymbol{A}^{b}=\left\langle\operatorname{Sign}^{b}, \mathrm{SEN}^{b}, N^{b}\right\rangle$. Consider the canonical pseudo-referential g-matrix system $\mathbb{A}=\langle\mathcal{A}, \mathcal{T}\rangle$ associated with $\mathcal{I}$, based on (PTS, $\mathcal{S}$ ), with $\mathcal{A}=\langle\boldsymbol{A},\langle F, \alpha\rangle\rangle$ and $\boldsymbol{A}=\left\langle\mathbf{S i g n}^{b}, \mathrm{SEN}, N\right\rangle$, as constructed in the proof of Theorem 5 .

Recall that the Frege equivalence system $\Lambda(\mathcal{I})=\left\{\Lambda_{\Sigma}(\mathcal{I})\right\}_{\Sigma \in|\operatorname{Sign}|}$ of $\mathcal{I}$ is defined, for all $\Sigma \in\left|\mathbf{S i g n}^{b}\right|$ and all $\varphi, \psi \in \operatorname{SEN}^{b}(\Sigma)$, by

$$
\langle\varphi, \psi\rangle \in \Lambda_{\Sigma}(\mathcal{I}) \quad \text { iff } \quad C_{\Sigma}(\varphi)=C_{\Sigma}(\psi) .
$$

By selfextensionality, $\Lambda(\mathcal{I})$ is a congruence system on $\boldsymbol{A}^{\mathrm{b}}$ and, in fact, coincides with the Tarski congruence system $\widetilde{\Omega}(\mathcal{I})$.

We define on the underlying algebraic system $\boldsymbol{A}=\left\langle\mathbf{S i g n}^{b}, \mathrm{SEN}, N\right\rangle$ of the canonical pseudo-referential g-matrix system $\mathbb{A}$ associated with $\mathcal{I}$ the relation family $\equiv^{\mathcal{I}}=\left\{\equiv_{\Sigma}^{\mathcal{I}}\right\}_{\Sigma \in \mid \mathbf{S i g n}^{b}}$, by setting, , for all $\Sigma \in\left|\mathbf{S i g n}^{b}\right|$ and all $\varphi, \psi \in \operatorname{SEN}^{b}(\Sigma)$,

$$
\{\varphi\} \equiv_{\Sigma}^{\mathcal{I}}\{\psi\} \quad \text { iff } \quad\langle\varphi, \psi\rangle \in \Lambda_{\Sigma}(\mathcal{I}) .
$$

Clearly, $\equiv^{\mathcal{I}}$ is an equivalence family on $\boldsymbol{A}$. Moreover, it is an equivalence system because of structurality. This establishes that the quotient functor $\mathrm{SEN}^{\Xi^{\mathcal{I}}}:=\mathrm{SEN} / \equiv^{\mathcal{I}}: \mathbf{S i g n}^{b} \rightarrow$ Set is well-defined (see [13]). 
Note that $\mathrm{SEN}^{\Xi^{\mathcal{I}}}$ may be considered as a point-based functor, based on $\operatorname{Th}(\mathcal{I})=\left\{\operatorname{Th}_{\Sigma}(\mathcal{I})\right\}_{\Sigma \in\left|\mathbf{S i g n}^{b}\right|}$ under the identification

$$
\{\varphi\} / \equiv_{\Sigma}^{\mathcal{I}} \longleftrightarrow \operatorname{Th}_{\Sigma}(\varphi)
$$

for all $\varphi \in \operatorname{SEN}^{b}(\Sigma), \Sigma \in\left|\mathbf{S i g n}^{b}\right|$ (which is well-defined by the definition of $\left.\equiv^{\mathcal{I}}\right)$.

Next, observe that, by the self-extensionality of $\mathcal{I}$, the equivalence system $\equiv^{\mathcal{I}}$ is actually a congruence system on $\boldsymbol{A}$. In fact, for all $\sigma^{b}$ : $\left(\mathrm{SEN}^{b}\right)^{k} \rightarrow \mathrm{SEN}^{b}$ in $N^{b}$, for all $\Sigma \in\left|\mathbf{S i g n}^{b}\right|$ and all $\varphi_{0}, \psi_{0}, \ldots, \varphi_{k-1}, \psi_{k-1} \in$ $\operatorname{SEN}^{b}(\Sigma)$, such that $\left\{\varphi_{i}\right\} \equiv_{\Sigma}^{\mathcal{I}}\left\{\psi_{i}\right\}$, for all $i<k$, we get that $C_{\Sigma}\left(\varphi_{i}\right)=$ $C_{\Sigma}\left(\psi_{i}\right)$, for all $i \in I$, whence by self-extensionality, $C_{\Sigma}\left(\sigma_{\Sigma}^{b}\left(\varphi_{0}, \ldots, \varphi_{k-1}\right)=\right.$ $C_{\Sigma}\left(\sigma_{\Sigma}^{b}\left(\psi_{0}, \ldots, \psi_{k-1}\right)\right)$, giving that $\left\{\sigma_{\Sigma}^{b}\left(\varphi_{0}, \ldots, \varphi_{k-1}\right)\right\} \equiv_{\Sigma}^{\mathcal{I}}\left\{\sigma_{\Sigma}^{b}\left(\psi_{0}, \ldots, \psi_{k-1}\right)\right\}$. But, by the definition of $\sigma: \mathrm{SEN}^{k} \rightarrow \mathrm{SEN}$, the latter is equivalent to $\sigma_{\Sigma}\left(\left\{\varphi_{0}\right\}, \ldots,\left\{\varphi_{k-1}\right\}\right) \equiv_{\Sigma}^{\mathcal{I}} \sigma_{\Sigma}\left(\left\{\psi_{0}\right\}, \ldots,\left\{\psi_{k-1}\right\}\right)$.

Now we conclude that the quotient $\boldsymbol{A}^{\equiv^{\mathcal{I}}}:=\boldsymbol{A} / \equiv^{\mathcal{I}}=\left\langle\operatorname{Sign}^{b}, \mathrm{SEN}^{\equiv^{\mathcal{I}}}, N^{\equiv^{\mathcal{I}}}\right\rangle$ is a well-defined $N^{b}$-algebraic system.

Finally, recall that $\mathcal{T}=\left\{T^{i}: i \in I\right\}$, with $T^{i}=\left\{T_{\Sigma}^{i}\right\}_{\Sigma \in|\operatorname{Sign}|}$ given, for all $i \in I$ and all $\Sigma \in|\mathbf{S i g n}|$, by

$$
T_{\Sigma}^{i}=\left\{\{\varphi\} \in \operatorname{SEN}(\Sigma): \varphi \in S_{\Sigma}^{i}\right\}
$$

We note that $\equiv^{\mathcal{I}}$ is compatible with $T^{i}$, for all $i$, and, therefore, it is a (g-matrix) congruence system of $\mathbb{A}=\langle\mathcal{A}, \mathcal{T}\rangle$. In fact, for all $\Sigma \in\left|\mathbf{S i g n}^{b}\right|$ and all $\varphi, \psi \in \operatorname{SEN}^{b}(\Sigma)$, such that $\{\varphi\} \equiv_{\Sigma}^{\mathcal{I}}\{\psi\}$ and $\{\varphi\} \in T_{\Sigma}^{i}$, we get that $C_{\Sigma}(\varphi)=C_{\Sigma}(\psi)$ and $\varphi \in S_{\Sigma}^{i} \in \operatorname{Th}_{\Sigma}(\mathcal{I})$. Hence, we obtain $\psi \in S_{\Sigma}^{i}$, which shows that $\{\psi\} \in T_{\Sigma}^{i}$.

It follows that the quotient g-matrix system $\mathbb{A}^{\equiv^{\mathcal{I}}}=\left\langle\mathcal{A}^{\equiv^{\mathcal{I}}}, \mathcal{T}^{\equiv^{\mathcal{I}}}\right\rangle$ is welldefined.

To establish the equivalence of the canonical referential g-matrix system associated with $\mathcal{I}$ with the quotient $\mathbb{A}^{\Xi^{\mathcal{I}}}$ of the canonical pseudo-referential g-matrix system $\mathbb{A}$ associated with $\mathcal{I}$ it suffices to note that the mapping

$$
\operatorname{Th}_{\Sigma}(\varphi) \mapsto\{\varphi\} / \equiv \sum_{\Sigma}^{\mathcal{I}}
$$

for all $\Sigma \in\left|\mathbf{S i g n}^{b}\right|, \varphi \in \operatorname{SEN}^{b}(\Sigma)$, determines an isomorphism between these two g-matrix systems. 


\section{Discrete Pseudo-Referential Matrix Systems}

Let $\boldsymbol{A}^{\mathrm{b}}=\left\langle\mathbf{S i g n}^{\mathrm{b}}, \mathrm{SEN}^{\mathrm{b}}, N^{\mathrm{b}}\right\rangle$ be an algebraic system and $\mathbb{A}=\langle\mathcal{A}, \mathcal{T}\rangle$ a pseudo-referential g-matrix system relative to some $(\mathrm{PTS}, \mathcal{S})$, with $\mathcal{S}=$ $\left\{S^{i}: i \in I\right\}$, i.e., such that $\mathcal{T}=\left\{T^{i}: i \in I\right\}$, with

$$
T_{\Sigma}^{i}=\left\{X \in \operatorname{SEN}(\Sigma): X \cap S_{\Sigma}^{i} \neq \varnothing\right\},
$$

for all $\Sigma \in \mid$ Sign $\mid$ and all $i \in I$.

The pseudo-referential g-matrix system $\mathbb{A}$ will be called discrete if, for all $i \in I$, there exists $\Sigma_{i} \in|\mathbf{S i g n}|$, such that, for all $\Sigma \in|\mathbf{S i g n}|$,

$$
S_{\Sigma}^{i} \begin{cases}\epsilon\left\{\{p\}: p \in \operatorname{PTS}\left(\Sigma_{i}\right)\right\}, & \text { if } \Sigma=\Sigma_{i}, \\ =\varnothing, & \text { otherwise. }\end{cases}
$$

In this section, taking after the work of Marek [9], we show that every $\pi$-institution $\mathcal{I}=\left\langle\boldsymbol{A}^{\mathrm{b}}, C\right\rangle$ has a strongly adequate discrete pseudo-referential matrix system semantics. This is done by exhibiting, for every g-matrix system, an equivalent discrete pseudo-referential g-matrix system.

THEOREM 6. Let $\boldsymbol{A}^{b}=\left\langle\mathbf{S i g n}^{b}, \mathrm{SEN}^{b}, N^{b}\right\rangle$ be an algebraic system. For every $N^{b}$-g-matrix system $\mathbb{A}^{\#}=\left\langle\mathcal{A}^{\#}, \mathcal{T}^{\#}\right\rangle$, with $\mathcal{A}^{\#}=\left\langle\boldsymbol{A}^{\#},\left\langle F^{\#}, \alpha^{\#}\right\rangle\right\rangle$, $\boldsymbol{A}^{\#}=\left\langle\mathbf{S i g n}^{\#}, \mathrm{SEN}^{\#}, N^{\#}\right\rangle$, there exists a discrete pseudo referential g-matrix system $\mathbb{A}=\langle\mathcal{A}, \mathcal{T}\rangle$ relative to some $(\mathrm{PTS}, \mathcal{S})$, such that $\mathcal{I}^{\mathbb{A}}=\mathcal{I}^{\mathbb{A}^{\#}}$.

Proof: Let $\boldsymbol{A}^{b}=\left\langle\mathbf{S i g n}^{b}, \mathrm{SEN}^{b}, N^{b}\right\rangle$ be an algebraic system. Consider an $N^{b}$-g-matrix system $\mathbb{A}^{\#}=\left\langle\mathcal{A}^{\#}, \mathcal{T}^{\#}\right\rangle$, with $\mathcal{A}^{\#}=\left\langle\boldsymbol{A}^{\#},\left\langle F^{\#}, \alpha^{\#}\right\rangle\right\rangle, \boldsymbol{A}^{\#}=$ $\left\langle\operatorname{Sign}^{\#}, \mathrm{SEN}^{\#}, N^{\#}\right\rangle$ and $\mathcal{T}^{\#}=\left\{T^{\# i}: i \in I\right\}$.

For all $\Sigma \in\left|\mathbf{S i g n}^{\#}\right|$, consider a collection $\left\{x_{\Sigma}^{i}: i \in I\right\}$, where, for all $i \in I, x_{\Sigma}^{i} \notin \operatorname{SEN}^{\#}(\Sigma)$ and, for all $i, j \in I$, with $i \neq j, x_{\Sigma}^{i} \neq x_{\Sigma}^{j}$.

Now define

$$
\operatorname{PTS}(\Sigma)=\operatorname{SEN}^{\#}(\Sigma) \cup\left\{x_{\Sigma}^{i}: i \in I\right\} \text {, for all } \Sigma \in\left|\operatorname{Sign}^{\#}\right| .
$$

Moreover, let $\mathcal{S}=\left\{S^{\Sigma, i}: \Sigma \in\left|\mathbf{S i g n}^{\#}\right|, i \in I\right\}$, where, for all $\Sigma \in\left|\mathbf{S i g n}^{\#}\right|$ and all $i \in I, S^{\Sigma, i}=\left\{S_{\Sigma^{\prime}}^{\Sigma, i}\right\}_{\Sigma^{\prime} \in\left|\mathbf{S i g n}^{\#}\right|}$ is defined by setting

$$
S_{\Sigma^{\prime}}^{\Sigma, i}=\left\{\begin{array}{ll}
\left\{x_{\Sigma}^{i}\right\}, & \text { if } \Sigma^{\prime}=\Sigma \\
\varnothing, & \text { if } \Sigma^{\prime} \neq \Sigma
\end{array} \text {, for all } \Sigma^{\prime} \in\left|\mathbf{S i g n}^{\#}\right| .\right.
$$


Next, define, for all $\Sigma \in\left|\operatorname{Sign}^{\#}\right|$ and all $\varphi \in \operatorname{SEN}^{\#}(\Sigma), X_{\varphi} \subseteq \operatorname{PTS}(\Sigma)$, by

$$
p \in X_{\varphi} \Leftrightarrow p=\varphi \text { or }(\exists i \in I)\left(p=x_{\Sigma}^{i} \text { and } \varphi \in T_{\Sigma}^{\#, i}\right) .
$$

Claim: For all $\Sigma \in\left|\operatorname{Sign}^{\#}\right|$ and all $\varphi, \psi \in \operatorname{SEN}^{\#}(\Sigma), X_{\varphi}=X_{\psi}$ if and only if $\varphi=\psi$.

Proof of the Claim: The "if" direction is obvious. For the "only if", reasoning by contraposition, we note that if $\varphi \neq \psi$, then $\varphi \in X_{\varphi}$, whereas $\varphi \notin X_{\psi}$. Therefore $X_{\varphi} \neq X_{\psi}$.

Now define, for all $\Sigma \in\left|\mathbf{S i g n}^{\#}\right|$,

$$
\operatorname{SEN}(\Sigma)=\left\{X_{\varphi}: \varphi \in \operatorname{SEN}^{\#}(\Sigma)\right\}
$$

and, moreover, for all $\Sigma, \Sigma^{\prime} \in\left|\mathbf{S i g n}^{\#}\right|$ and all $f \in \operatorname{Sign}^{\#}\left(\Sigma, \Sigma^{\prime}\right)$, let $\operatorname{SEN}(f)$ : $\operatorname{SEN}(\Sigma) \rightarrow \operatorname{SEN}\left(\Sigma^{\prime}\right)$ be given, for all $\varphi \in \operatorname{SEN}^{\#}(\Sigma)$, by

$$
\operatorname{SEN}(f)\left(X_{\varphi}\right)=X_{\mathrm{SEN} \#(f)(\varphi)} .
$$

The fact that SEN : Sign ${ }^{\#} \rightarrow$ Set, thus defined, is a functor follows from the fact that $\mathrm{SEN}^{\#}$ is a functor.

Next, for all $\sigma:\left(\mathrm{SEN}^{b}\right)^{k} \rightarrow \mathrm{SEN}^{b}$ in $N^{b}$, we define $\sigma: \mathrm{SEN}^{k} \rightarrow$ SEN by letting, for all $\Sigma \in\left|\mathbf{S i g n}^{\#}\right|, \sigma_{\Sigma}: \operatorname{SEN}(\Sigma)^{k} \rightarrow \operatorname{SEN}(\Sigma)$ be given by

$$
\sigma_{\Sigma}\left(X_{\varphi_{0}}, \ldots, X_{\varphi_{k-1}}\right)=X_{\sigma_{\Sigma}^{\#}\left(\varphi_{0}, \ldots, \varphi_{k-1}\right)},
$$

for all $\varphi_{0}, \ldots, \varphi_{k-1} \in \operatorname{SEN}^{\#}(\Sigma)$.

This is well-defined by the preceding claim and, moreover, it is a bona fide natural transformation, since, for all $\Sigma, \Sigma^{\prime} \in\left|\mathbf{S i g n}^{\#}\right|, f \in \operatorname{Sign}^{\#}\left(\Sigma, \Sigma^{\prime}\right)$ and all $\varphi_{0}, \ldots, \varphi_{k-1} \in \operatorname{SEN}^{\#}(\Sigma)$, we have according to the preceding definitions,

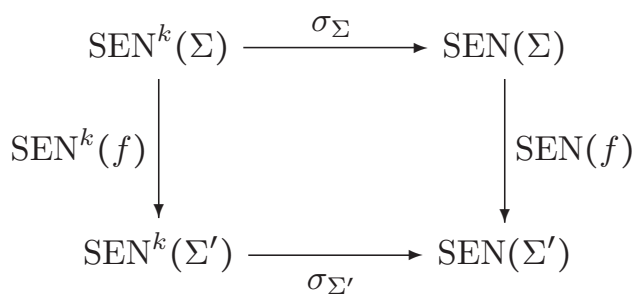




$$
\begin{aligned}
\operatorname{SEN}(f)\left(\sigma _ { \Sigma } \left(X_{\varphi_{0}}\right.\right. & \left.\left., \ldots, X_{\varphi_{k-1}}\right)\right) \\
& =\operatorname{SEN}(f)\left(X_{\sigma_{\Sigma}^{\#}\left(\varphi_{0}, \ldots, \varphi_{k-1}\right)}\right) \\
& =X_{\operatorname{SEN}^{\#}(f)\left(\sigma_{\Sigma}^{\#}\left(\varphi_{0}, \ldots, \varphi_{k-1}\right)\right)} \\
& =X_{\sigma_{\Sigma^{\prime}}^{\#}\left(\operatorname{SEN}^{\#}(f)\left(\varphi_{0}\right), \ldots, \operatorname{SEN}^{\#}(f)\left(\varphi_{k-1}\right)\right)} \\
& =\sigma_{\Sigma^{\prime}}\left(X_{\mathrm{SEN}^{\#}}(f)\left(\varphi_{0}\right), \ldots, X_{\mathrm{SEN}^{\#}}(f)\left(\varphi_{k-1}\right)\right. \\
& =\sigma_{\Sigma^{\prime}}\left(\operatorname{SEN}(f)\left(X_{\varphi_{0}}, \ldots, X_{\varphi_{k-1}}\right)\right) .
\end{aligned}
$$

Let $N$ be the category consisting of all natural transformations $\sigma$, for $\sigma^{\#}$ in $N^{\#}$. Then the triple $\boldsymbol{A}=\left\langle\mathbf{S i g n}^{\#}, \mathrm{SEN}, N\right\rangle$ is a referential $N^{b}$-algebraic system.

Define $\langle F, \alpha\rangle: \boldsymbol{A}^{b} \rightarrow \boldsymbol{A}$ as follows:

- $F: \mathbf{S i g n}^{b} \rightarrow \mathbf{S i g n}^{\#}$ is equal to $F^{\#}: \mathbf{S i g n}^{b} \rightarrow \mathbf{S i g n}^{\#}$;

- $\alpha: \mathrm{SEN}^{b} \rightarrow \mathrm{SEN} \circ F$ is defined by letting, for all $\Sigma \in\left|\mathbf{S i g n}^{b}\right|, \alpha_{\Sigma}$ : $\operatorname{SEN}^{b}(\Sigma) \rightarrow \operatorname{SEN}(F(\Sigma))$ be given by

$$
\alpha_{\Sigma}(\varphi)=X_{\alpha_{\Sigma}^{\#}(\varphi)}, \quad \text { for all } \varphi \in \operatorname{SEN}^{\#}(\Sigma) .
$$

Again this definition makes $\alpha: \operatorname{SEN}^{b} \rightarrow$ SEN $\circ F$ a bona fide natural transformation, since, for all $\Sigma, \Sigma^{\prime} \in\left|\operatorname{Sign}^{b}\right|$, all $f \in \operatorname{Sign}^{b}\left(\Sigma, \Sigma^{\prime}\right)$ and all $\varphi \in \operatorname{SEN}^{b}(\Sigma)$, we have

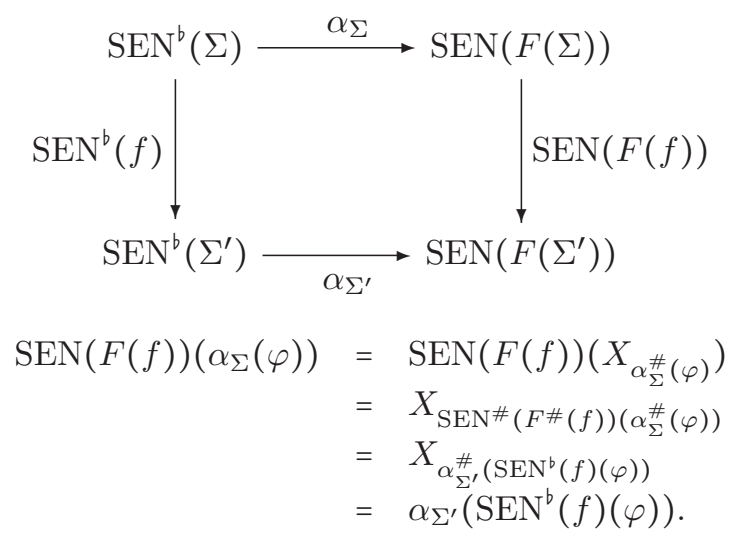

Moreover, $\langle F, \alpha\rangle: \boldsymbol{A}^{b} \rightarrow \boldsymbol{A}$ is an algebraic system morphism. Indeed, for all $\sigma^{b}:\left(\operatorname{SEN}^{b}\right)^{k} \rightarrow \operatorname{SEN}^{b}$ in $N^{b}$, all $\Sigma \in\left|\operatorname{Sign}^{b}\right|$ and all $\varphi_{0}, \ldots, \varphi_{k-1} \in \operatorname{SEN}^{b}(\Sigma)$, we have 


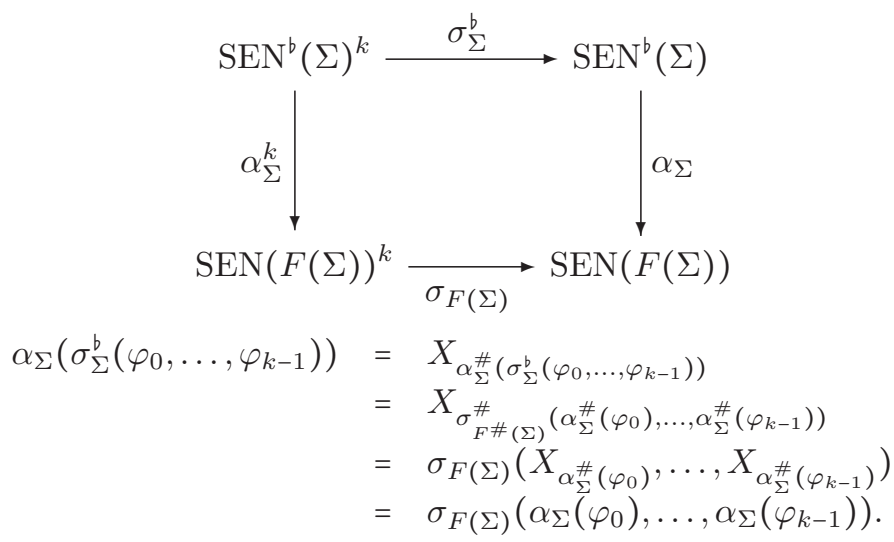

Thus, the pair $\mathcal{A}=\langle\boldsymbol{A},\langle F, \alpha\rangle\rangle$ is an interpreted referential $N^{\mathrm{b}}$-algebraic system.

Let $\mathbb{A}=\langle\mathcal{A}, \mathcal{T}\rangle$ be the discrete pseudo-referential $N^{\mathrm{b}}$-g-matrix system relative to (PTS, $\mathcal{S}$ ), where $\mathcal{S}=\left\{S^{\Sigma, i}: \Sigma \in\left|\mathbf{S i g n}^{\#}\right|, i \in I\right\}$, as before, with $\mathcal{T}^{\#}=\left\{T^{\# i}: i \in I\right\}$ being the collection of filter families of the g-matrix system $\mathbb{A}^{\#}$.

Then, for all $i \in I$ and for all $\Sigma \in\left|\mathbf{S i g n}^{\#}\right|$, we have $T^{\Sigma, i}=\left\{T_{\Sigma^{\prime}}^{\Sigma, i}\right\}_{\Sigma^{\prime} \epsilon\left|\mathbf{S i g n}^{\#}\right|}$, where, for all $\Sigma^{\prime} \in\left|\mathbf{S i g n}^{\#}\right|$,

$$
\begin{aligned}
T_{\Sigma^{\prime}}^{\Sigma, i} & =\left\{X \in \operatorname{SEN}\left(\Sigma^{\prime}\right): X \cap S_{\Sigma^{\prime}}^{\Sigma, i} \neq \varnothing\right\} \\
& = \begin{cases}\varnothing, & \text { if } \Sigma^{\prime} \neq \Sigma, \\
\left\{X_{\varphi}: x_{\Sigma}^{i} \in X_{\varphi}, \varphi \in \operatorname{SEN}^{\#}(\Sigma)\right\}, & \text { if } \Sigma^{\prime}=\Sigma\end{cases} \\
& = \begin{cases}\varnothing, & \text { if } \Sigma^{\prime} \neq \Sigma \\
\left\{X_{\varphi}: \varphi \in T_{\Sigma}^{\# i}\right\}, & \text { if } \Sigma^{\prime}=\Sigma\end{cases}
\end{aligned}
$$

Now notice that, for all $\Sigma \in\left|\operatorname{Sign}^{b}\right|$ and all $\varphi \in \operatorname{SEN}^{b}(\Sigma)$, we have that, for all $\Sigma^{\prime} \in\left|\mathbf{S i g n}^{b}\right|$, all $f \in \operatorname{Sign}^{b}\left(\Sigma, \Sigma^{\prime}\right)$ and all $i \in I$,

$$
\alpha_{\Sigma^{\prime}}\left(\operatorname{SEN}^{b}(f)(\varphi)\right) \in T_{F\left(\Sigma^{\prime}\right)}^{F\left(\Sigma^{\prime}\right), i} \quad \text { iff } \quad \alpha_{\Sigma^{\prime}}^{\#}\left(\operatorname{SEN}^{b}(f)(\varphi)\right) \in T_{F\left(\Sigma^{\prime}\right)}^{\# i} .
$$

Equation (7.1) is true because, from the expression obtained from $T^{\Sigma, i}$ above, we obtain

$$
\begin{array}{lll}
\alpha_{\Sigma^{\prime}}\left(\operatorname{SEN}^{b}(f)(\varphi)\right) \in T_{F\left(\Sigma^{\prime}\right), i}^{F\left(\Sigma^{\prime}\right),} \text { iff } & X_{\alpha_{\Sigma^{\prime}}^{\#}\left(\operatorname{SEN}^{b}(f)(\varphi)\right)} \in\left\{X_{\varphi}: \varphi \in T_{F\left(\Sigma^{\prime}\right)}^{\# i}\right\} \\
& \text { iff } \quad \alpha_{\Sigma^{\prime}}^{\#}\left(\operatorname{SEN}^{b}(f)(\varphi)\right) \in T_{F\left(\Sigma^{\prime}\right)}^{\# i} .
\end{array}
$$


Finally, we get the desired conclusion expressed in the following

Claim: $\mathcal{I}^{\mathbb{A}}=\mathcal{I}^{\mathbb{A}^{\#}}$.

Let $\Sigma \in\left|\operatorname{Sign}^{b}\right|$ and $\Phi \cup\{\varphi\} \subseteq \operatorname{SEN}^{b}(\Sigma)$. Then we have $\varphi \in C_{\Sigma}^{\mathbb{A}}(\Phi)$ iff, for all $\Sigma^{\prime} \in\left|\mathbf{S i g n}^{b}\right|$, all $f \in \operatorname{Sign}^{b}\left(\Sigma, \Sigma^{\prime}\right)$ and all $i \in I$,

$$
\alpha_{\Sigma^{\prime}}\left(\operatorname{SEN}^{b}(f)(\Phi)\right) \subseteq T_{F\left(\Sigma^{\prime}\right)}^{F\left(\Sigma^{\prime}\right), i} \quad \text { implies } \quad \alpha_{\Sigma^{\prime}}\left(\operatorname{SEN}^{b}(f)(\varphi)\right) \in T_{F\left(\Sigma^{\prime}\right)}^{F\left(\Sigma^{\prime}\right), i},
$$

iff, by Equivalence (7.1), for all $\Sigma^{\prime} \in\left|\operatorname{Sign}^{b}\right|$, all $f \in \operatorname{Sign}^{b}\left(\Sigma, \Sigma^{\prime}\right)$ and all $i \in I$,

$$
\alpha_{\Sigma^{\prime}}^{\#}\left(\operatorname{SEN}^{b}(f)(\Phi)\right) \subseteq T_{F\left(\Sigma^{\prime}\right)}^{\# i} \quad \text { implies } \quad \alpha_{\Sigma^{\prime}}^{\#}\left(\operatorname{SEN}^{b}(f)(\varphi)\right) \in T_{F\left(\Sigma^{\prime}\right)}^{\# i},
$$

iff $\varphi \in C_{\Sigma}^{\mathbb{A}^{\#}}(\Phi)$. Since $C^{\mathbb{A}}=C^{\mathbb{A}^{\#}}$, we conclude that $\mathcal{I}^{\mathbb{A}}=\mathcal{I}^{\mathbb{A}^{\#}}$, as required.

\section{Acknowledgements}

This work owes its existence to the pioneering work of Wójcicki, Malinowski and Marek. The author acknowledges the scientific debt owed to the works of these logicians. The author has a lasting personal and professional debt to Don Pigozzi, who introduced him to algebraic logic in the mid 1990's and has been a source of inspiration and has provided firm support ever since.

\section{References}

[1] W. J. Blok D. and Pigozzi, Algebraizable Logics, Memoirs of the American Mathematical Society, Vol. 77, No. 396 (1989).

[2] J. Czelakowski, Reduced Products of Logical Matrices, Studia Logica, Vol. 39 (1980), pp. 19-43.

[3] J. Czelakowski, The Suszko Operator Part I, Studia Logica, Vol. 74, No. 1-2 (2003), pp. 181-231.

[4] J. Fiadeiro and A. Sernadas, Structuring Theories on Consequence, [in:] D. Sannella and A. Tarlecki, eds., Recent Trends in Data Type Specification, Lecture Notes in Computer Science, Vol. 332, Springer-Verlag, New York, 1988, pp. 44-72.

[5] J. M. Font and R. Jansana, A General Algebraic Semantics for Sentential Logics, Lecture Notes in Logic, Vol. 332, No. 7 (1996), Springer-Verlag, Berlin Heidelberg, 1996. 
[6] J. A. Goguen and R. M. Burstall, Institutions: Abstract Model Theory for Specification and Programming, Journal of the Association for Computing Machinery, Vol. 39, No. 1 (1992), pp. 95-146.

[7] R. Jansana and A. Palmigiano, Referential Semantics: Duality and Applications, Reports on Mathematical Logic, Vol. 41 (2006), pp. 63-93.

[8] G. Malinowski, Pseudo-Referential Matrix Semantics for Propositional Logics, Bulletin of the Section of Logic, Vol. 12, No. 3 (1983), pp. 90-98.

[9] I. Marek, Remarks on Pseudo-Referential Matrices, Bulletin of the Section of Logic, Vol. 16, No. 2 (1987), pp. 89-92.

[10] R. Wójcicki, Referential Matrix Semantics for Propositional Calculi, Bulletin of the Section of Logic, Vol. 8, No. 4 (1979), pp. 170-176.

[11] G. Voutsadakis, Categorical Abstract Algebraic Logic: Referential Algebraic Semantics, Studia Logica, Vol. 101, No. 4 (2013), pp. 849-899.

[12] G. Voutsadakis, Categorical Abstract Algebraic Logic: Referential $\pi$-Institutions, Bulletin of the Section of Logic, Vol. 44, No. 1/2 (2015), pp. 33-51.

[13] G. Voutsadakis, Categorical Abstract Algebraic Logic: Tarski Congruence Systems, Logical Morphisms and Logical Quotients, Journal of Pure and Applied Mathematics: Advances and Applications, Vol. 13, No. 1 (2015), pp. 27-73.

School of Mathematics and Computer Science

Lake Superior State University

Sault Sainte Marie, MI 49783, USA,

e-mail: gvoutsad@lssu.edu 